Proc. Indian Acad. Sci. (Math. Sci.), Vol. 89, Number 2, May 1980, pp. 75-93. (C) Printed in India.

\title{
A cryptographic system based on finite field transforms
}

\author{
E V KRISHNAMURTHY and VLJAYA RAMACHANDRAN \\ School of Automation, Indian Institute of Science, Bangalore 560 012, Inciia
}

MS received 24 March 1979; revised 21 August 1979

\begin{abstract}
In this paper, we develop a cipher system based on finite field transforms. In this system, blocks of the input character-string are enciphered using congruence or modular transformations with respect to either primes or irreducible polynomials over a finite field. The polynomial system is shown to be clearly superior to the prime system for conventional cryptographic work.
\end{abstract}

Keywords. Ciphers; computer data protection; cryptography; finite field transforms; irreducible polynomials; military communication; polynomial congruences; prime congruences; public crypto-systems.

\section{Introduction}

A cryptographic system [7], [11] consists of a set of transformations $T_{j}$, each of which can act on an input mossage $M$ to produce a corresponding enciphered message $E_{i}$, i.e.,

$$
E_{j}=T_{1}(M) \text {. }
$$

Each transformation $T_{j}$ is spesified by an associated key $k_{j}$. The enciphered message is transmitted to the reseiver over an interceptable medium. At the receiving end, the original message $M$ is rezovered by applying the inverse transformation $T_{j}^{-1}$ on the reseived (en zip iered) message $E_{j}$. It is clear that the existence of $T_{j}^{-1}$ is a nesessary condition for $T_{j}$ to be a valid encoding transformation.

\section{Example :}

Substitution cipher-In this cipher, each input character is transformed into another character. The transformed character set may or may not be the same as the input character sel. In the former case, the transformation is a simple permutation of the input charaster set. Let the (ordered) input characters be $a, b, c, d$. If the transformed character set is $\{0,1,2,3\}$, any permutation of these 4 characters represents a key to a particular transformation. If $2,1,3,0$ is the specified key $k_{j}, T_{\text {j }}$ is given by $a \rightarrow 2, b \rightarrow 1, c \rightarrow 3, d \rightarrow 0$, pna $T_{j}^{-1}$ is given by $0 \rightarrow d, 1 \rightarrow b$, $2 \rightarrow a$, and $3 \rightarrow c$. There are $4 !=24$ different transformations possible.

It is assumed that the enemy (i.e., the persons from whom the message $M$ is protected) knows the set of transformations $T_{j}$ being used. He also has available, 